\title{
Multi-rope Hoist Steel Rope Tension On-line Monitoring System
}

\author{
Liu Jian \\ School of Information and Communication Engineering \\ Beijing University of Posts and Telecommunications \\ Beijing, China
}

\author{
Liu Yuan-xiang \\ Zhenghui corporation of Fenxi Mine Group \\ Shanxi Coking Coal Group CO.,LTD. \\ Jiexiu, China
}

\begin{abstract}
A multi-rope hoist steel rope tension on-line monitoring system is introduced in this paper, which consists of hydraulic sensors, data acquisition and sending devices, wireless communication devices and IPC. This system monitors the tension of multi-rope hoist steel ropes, in order to get the actual load of the hoist. When error happens the system can alarm to avoid accidents, such as serious imbalance of the steel ropes and hoist overload.
\end{abstract}

Keywords- multi-rope hoist; steel rope; tension; on-line monitor; tension imbalance

\section{INTRODUCTION}

Multi-rope hoist is the main lifting device for coal mining in China. In the work process, it is easy to cause broken rope, cage falling and other accidents, since the hoist steel rope tension is too large ${ }^{[1]}$. Research shows that, the key reason for the large tension of hoist ropes is overload and large tension difference of the hoist ropes. According to "Coal mine Safety regulation", in multi-rope friction lifting device, any of the hoist ropes' tension difference and the average tension difference both should not exceed $\pm 10 \%{ }^{[2]}$. Therefore, it is of great significance to monitor the ropes' tension of multi-rope hoist and calculate the actual load of the hoist and tension difference of the ropes. The system can ensure coal mine safety production and extend the life of the hoist ropes and the friction pad.

In recent years, the tension monitoring methods for multirope hoist's rope have been studied in china and abroad. In abroad, the research in this field starts earlier and has obtained some achievements. However, the high price and strict environmental requirements make the foreign research results difficult to apply in Chinese mine hoist system. Compared with the foreign research, less research work has been done by domestic researchers in this field, and most of the monitoring methods are indirect and static, such as notation method, shock wave method and the pressure roller-power electric conversion sensor method. At present, some domestic scientific research institutions also do some research on dynamic monitoring methods for multi-rope hoist's rope, but the research results have little application in actual production. By studying on multi-rope hoist system and analyzing the reasons for tension imbalance according to the measured data, this paper proposes a multi-rope hoist steel rope tension on-line monitoring system.

\section{SENSOR SELECTION AND INSTALLATION}

Most of the multi-rope friction hoists install steel rope hydraulic tension automatic balancing device, which can regulate the tension of each steel rope through the balance cylinders connected with the steel ropes, to ensure the four steel ropes' tension approximately equal ${ }^{[3]}$. In the actual operation, the balance cylinders are set as one side open and the other side closed, because if the balance cylinders of the two skips both open may result in "rope skipping" phenomenon and accelerate the rope and friction pad wear and tear. In this condition, the tensions of the open side are balanced, while the closed side exists tension difference obviously. By installing hydraulic sensors on each balance cylinder, we can get the hydraulic pressures. The tension difference and the load of the hoist can be obtained by calculating and analyzing the pressures data. Hydraulic pressure sensors should be installed between the balance cylinder and spherical stop valve, and the on-site installation diagram is shown in Figure 1.

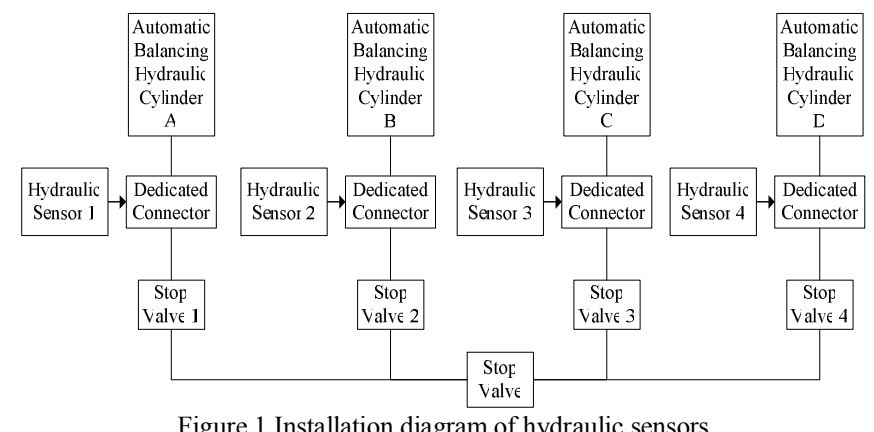

III. OVERALL MONITORING SCHEME

The monitoring system consists of hydraulic data acquisition and sending devices, wireless communication devices, skip position monitoring devices and IPC. Hydraulic data are uploaded to the wireless communication device installed in wellhead by the hydraulic data acquisition device and wireless communication module, which are powered by battery, and then the data are send to the IPC in hoist house via RS485 communication cable. The skip position monitoring device can monitor the position signal and arriving signal of the skip by installing magnetic steels and sensors on the hoist shaft and skip respectively, and send the data to IPC via serial port in real time. The IPC calculates and display the position of the skip, the tension differences and other data, according to the 
received data. If the hoist overloads or other errors happen, the system will be sound and light alarm, even cut the safety circuit by the relay in the skip position monitoring devices when necessary. The structure of the system is shown in Figure 2.

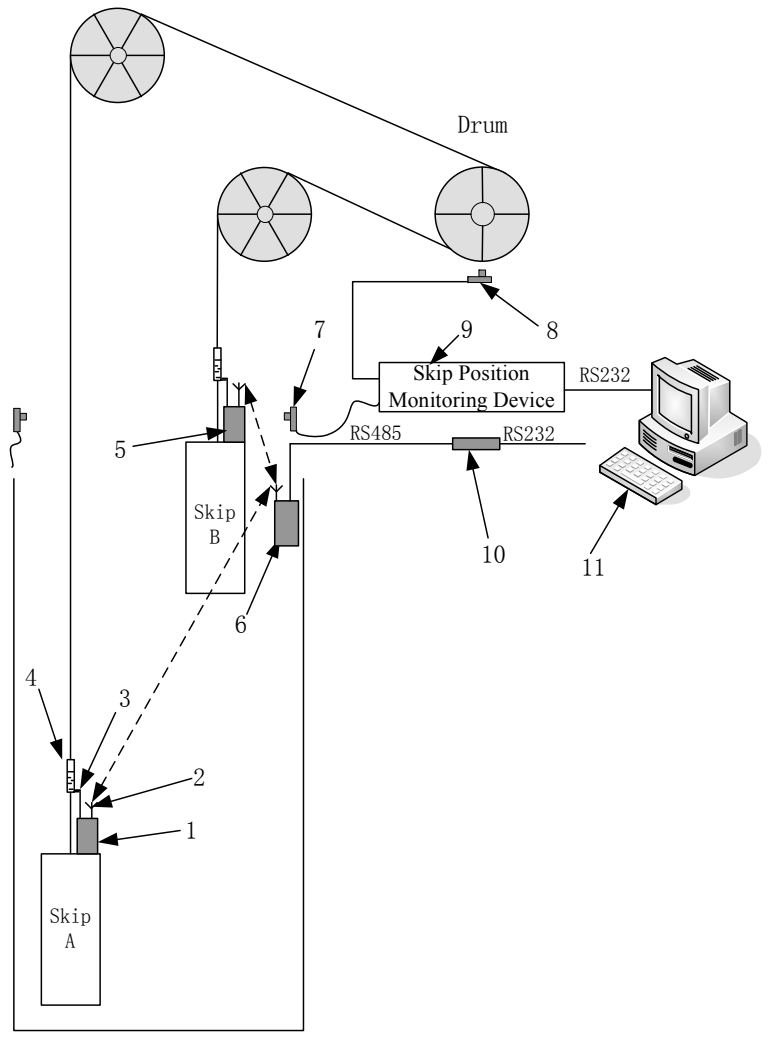

1-Data acquisition module A 2-Wireless communication module 3-Hydraulic sensor 4-Automatic balancing hydraulic cylinder 5-Data acquisition module $\mathrm{B} 6$-Wireless communication module in wellhead 7-Proximity switch

8-Hall sensor 9-Skip position monitoring device 10- RS485/232 converter $11-$ IPC

Figure 2 Structure of multi -rope hoist steel rope tension on-line monitoring system

\section{HYDRAULIC DATA ACQUISITION AND SENDING DEVICE}

The function of the hydraulic data acquisition and sending device is acquiring the hydraulic data, A/D conversion, packaging the converted data and sending them to the wireless receiving device. The acquisition and sending device is made up of data acquisition circuit, wireless communication module, battery and other modules.

The core of data acquisition circuit is AVR series microcontroller ATmega16L, which is of high performance. ATmega16L drives the 12-bit multi-channel digital converter TLC2543I to converter the hydraulic data, calculates and packages the data, and sends them to the wireless communication module. The circuit diagram is shown in Figure 3.

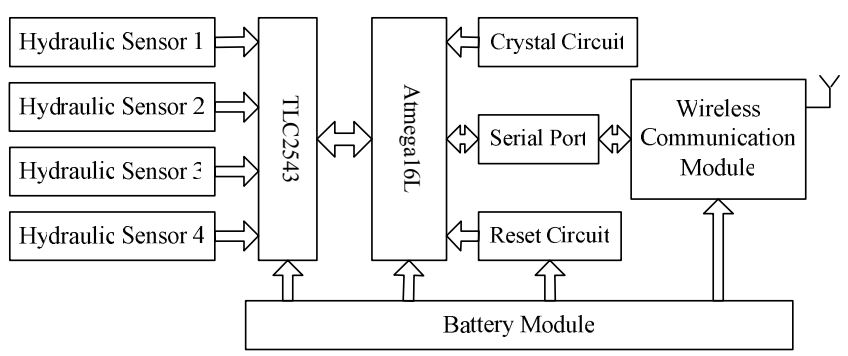

Figure 3 Circuit diagrams of hydraulic pressure data acquisition and sending device

\section{SKIP POSITION MONITORING DEVICE}

The skip position monitoring device is installed in the hoist house. It acquires the position signal of the skip in real time, and the main functions of it are as follows:

- Acquiring the arriving signal of the skip in real time so as to provide accurate signal for the system. The arriving signal not only can help to revise the position signal of the skip, but also provides time signal for IPC to diagnose faults.

- Calculating the position of the skip in real time. By installing magnetic steels on the hoist shaft and hall sensor aside, the system can get the speed and direction of the skip, and then calculates the exact position of the skip according to the principle of hall velocity measurement and ranging.

- Controlling the on-off state of the driving circuit. Once the monitoring parameters overrun, the device will alarm, even cut off the driving circuit to prevent accident when necessary.

\section{A. Skip arriving signal detection circuit}

Skip arriving signal is a switch signal that indicates whether the skip reaches the tipping point. The system needs to monitor two skip arriving signals since there are two skips, and the signals are measured by magnetic proximity switches. The magnetic proximity switch installation diagram and the skip arriving signal detection circuit are shown in Figure 4 and Figure 5 respectively.

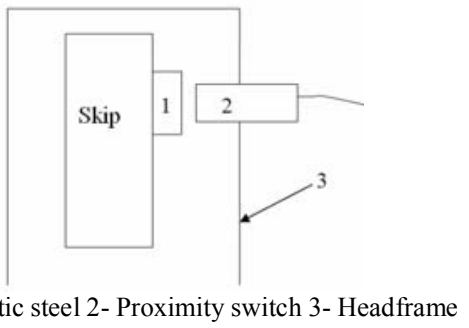

1- Magnetic steel 2- Proximity switch 3- Headframe

Figure 4 Magnetic proximity switch installation diagram 


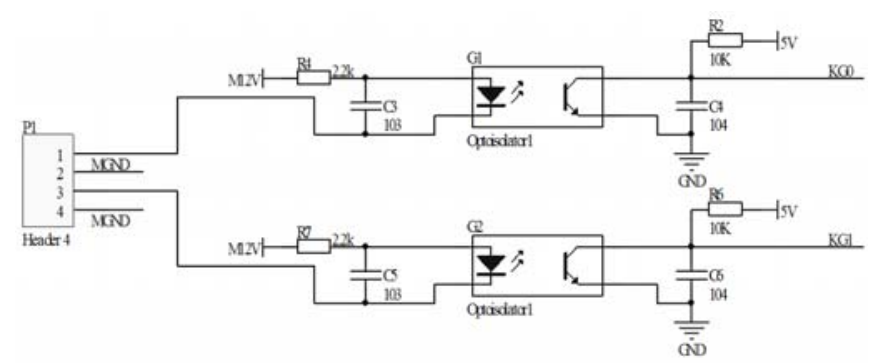

Figure 5 Skip arriving signal detection circuit

In Figure 5, G1 and G2 are optocouplers TLP521-1, used to isolate the arriving signals and the circuit. The port 1 and port 2 of $\mathrm{P} 1$ are connected with one arriving signal, and port 3 and port 4 are connected with another arriving signal. Port 1 and port 2 are connected with the two ports of the magnetic proximity switch. If the switch is closed, port 1 and port 2 will be shorted, which means port 2 will be set as low level. On this occasion, there is current flowing through the light-emitting diodes G1, conducting the emitter collector of G1, and then KG0 will be set as low by GND. On the contrary, if the switch is open, KG0 will be high level. The microcontroller reads the level of the KG0, judges the state of the magnetic proximity switch from the KG0 signal, and thus judges whether the skip has reached the tipping point.

\section{B. Monitoring circuit of hall sensors}

The steel rope tension on-line monitoring system monitors the position of the skip by installing magnetic steels on the hoist shaft and a hall sensor in the vicinity. We can obtain the direction and depth of the skip by measuring the number of pulses and the direction of the hoist roller when the hoist is working. The hall speed sensor is of high accuracy, and its output signal can be transmitted and processed over a long distance. The installation diagram of hall sensor is shown in Figure 6.

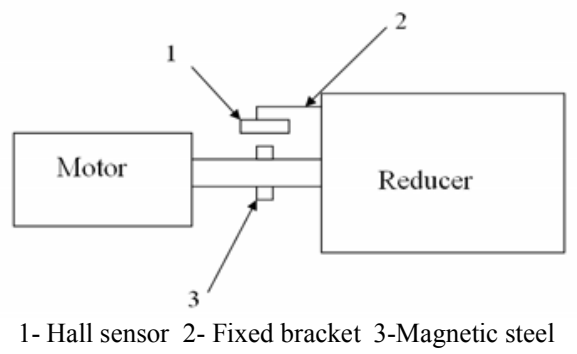

Figure 6 Installation diagram of hall sensor

The dual-hall sensor detection circuit is shown in Figure 7. Port 1 , port 2 and port 3 of P5 are connected with hall sensor 1 , and the port 4 , port 5 and port 6 are connected with hall sensor 2. The detection principle of hall sensor is the same as the skip arriving signal. In the figure, G5 and G6 are optocouplers TLP521-1, used to isolate the pulse signal and the circuit. Port 1 , port 2 and port 3 are connected with the power port, signal port, and GND port respectively. If the magnetic steel close to the hall sensor, the port 2 and port 3 will be connected directly, which means port 2 will be set as low level. On this occasion, there is current flowing through the light-emitting diodes in G5, conducting the emitter collector of G5, and then IPC will be set as low by GND. On the contrary, if magnetic steel is away from the hall sensor, KG0 will be high level. The microcontroller obtains the direction and turns of rolls by comparing the state of ICP and SIN, and then calculates the position of the skip.

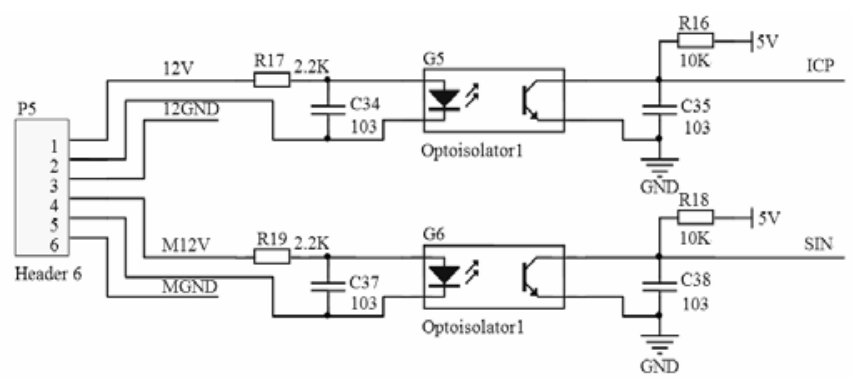

Figure 7 Dual-hall sensor detection circuit

\section{ALGORITHM OF THE TENSION MONITORING}

The fault type can be judged according to the tension data and the position of the skip, such as overload, tension imbalance of the steel ropes, unloading coal uncompleted, weighting imbalance and hydraulic cylinder compressed to the limit. Except for the tension data and the position of the skip, the load of the skip in wellhead and shaft bottom when no-load must be record. Once the equipment installation is completed, the IPC should record the loads $Q_{\mathrm{d}}$ and $Q_{\mathrm{u}}$ in wellhead and shaft bottom when no-load. The specific fault judging methods are as follows:

\section{A. Overload}

The actual load of the skip after loading $Q_{\mathrm{s}}$ pluses the load when no-load equals the net load of the hoist $Q_{z}$.

$$
Q_{\mathrm{z}}=Q_{\mathrm{s}}-Q_{\mathrm{d}}
$$

In the course of loading, the impact force caused by coal dropping will lead to $Q_{z}$ greater than the true load, so overload must be detected when loading finished. If the net load exceeds the maximum allowable loading coal, the system estimates that the hoist is overload.

\section{B. Tension imbalance:}

The real-time tensions of the steel four ropes are $F_{1}, F_{2}$, $F_{3}, F_{4}$. According to the "Coal Mine Safety Regulation", the formula for calculating the tension imbalance degree of the steel rope is as follow ${ }^{[4]}$.

$$
B=\frac{\max \left|F_{i}-\frac{1}{4} \sum_{i=1}^{4} F_{i}\right|}{\frac{1}{4} \sum_{i=1}^{4} F_{i}} \times 100 \%
$$


If the monitored tension imbalance degree is greater than $10 \%$, the system will alarm as tension imbalance of the steel ropes.

\section{Unloading coal uncompleted}

Before detecting the tension signals, there is a fivesecond delay just the skip arrives at the wellhead, to wait for unloading is completed. The amount of the coal attached to the skip is the difference of the actual load after unloading $Q_{s}$ and the load when no-load in wellhead $Q_{u}$.

$$
Q_{\mathrm{n}}=Q_{\mathrm{s}}-Q_{\mathrm{u}}
$$

If the amount of the coal attached to the skip exceeds the specified value, the system will alarm as unloading coal uncompleted. When the skip leaves the wellhead, the fault of unloading coal uncompleted will not be detected.

\section{Hydraulic cylinder compressed to the limit}

When one of the hydraulic cylinders in hydraulic tension automatic balancing device in compressed to the limit, the tension of the steel rope will impact on the steel frame directly, that means the tension automatic balancing device is out of action. At this moment, the load calculated from hydraulic pressures is less than the actual load. When the skip in wellhead, if the actual load is less than the load when no$\operatorname{load} Q_{\mathrm{u}}$, that is

$$
Q_{\mathrm{s}}<Q_{\mathrm{u}}
$$

The system will alarm as hydraulic cylinder compressed to the limit.

\section{E. Weighting imbalance}

Weight difference is the difference between the actual loads of the two skips wellhead or shaft bottom. If the actual loads of the two skips are $Q_{\mathrm{za}}$ and $Q_{\mathrm{zb}}$ respectively, the weight difference can be calculated as

$$
Q_{\mathrm{j}}=\left|Q_{\mathrm{za}}-Q_{\mathrm{zb}}\right|
$$

"Coal Mine Safety Regulation" provides that the weight difference of the hoist should not exceed the specified value, otherwise the hoist may occur rope slipping accident.

\section{ANALYSIS OF THE EXPERIMENT DATA}

Figure 8 shows the tension curves of the main skip when the main skip rises and vice skip falls, and the Figure 9 shows the tension curves of the vice skip at the same time. The abscissa of tension curve is the position of the skip (the wellhead is $0 \mathrm{~m}$, and shaft bottom is $705 \mathrm{~m}$ ), the ordinate is the tensions of the four steel ropes corresponds to the position of the skip. From shaft bottom to wellhead, the tension has a tendency to increase, that is because the tail rope of the hoist weights about 20 tons. As we mentioned in Part 1, the balance cylinders are set as one side open and the other side closed. The tension difference of main skip is obviously since the balance cylinders of main skip are closed, while the tension curves of the four steel ropes of vice skip coincide approximately since its balance cylinders are open.

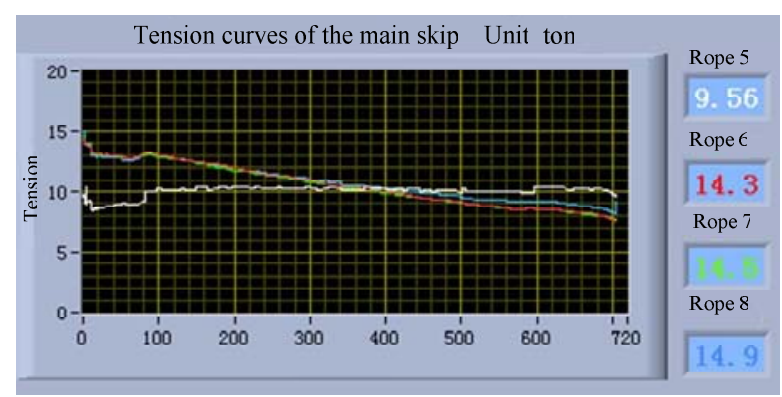

Figure 8 Tension curves of the main skip

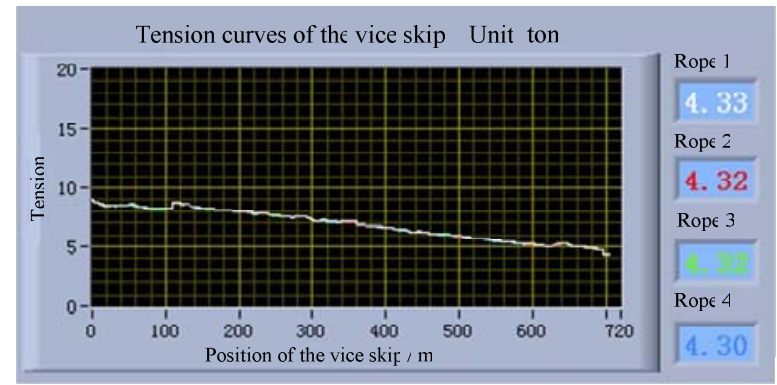

Figure 9 Tension curves of the vice skip

\section{CONCLUSION}

A steel rope tension on-line monitoring system for multirope hoist is proposed in this paper, which measures the hydraulic data by installing hydraulic pressure sensors on the hydraulic tension automatic balancing device. The hydraulic data acquisition and sending devices on the skip acquires the hydraulic data and sends it to the IPC, to realize the on-line monitor the tension of the steel ropes. The experiment shows that the system runs stable, and the data obtained are accurate and reliable.

\section{REFERENCES}

[1] Yao Wen-bin, Shao Qian-jun and Zhang Wei, "Study on mine hoist wire rope tension meter". Journal of China coal society, vol.29, no.3, pp.371375, 2004. ( in Chinese)

[2] Administration of work safety and state administration of coal mine safety. Coal mine safety regulations, Beijing: China coal industry publishing house, 2005. ( in Chinese)

[3] Wang Jin-feng, Lan Jian-zeng and Wei Xiao-yan. "Measurement of the tension difference for multi-rope hoist steel rope", Journal of energy technology and management, vol.32, no.6, pp.94-95, 2007.( in Chinese)

[4] Zhang Hua-zhong. Research on checking and processing of lift wireRope tension signals base on wireless transmission, Master dissertation, Shandong University of Science and technology, Jinan, China, 2006. 